Pacific Journal of Mathematics

ISOPERIMETRIC RATIOS OF REULEAUX POLYGONS 


\title{
ISOPERIMETRIC RATIOS OF REULEAUX POLYGONS
}

\author{
W. J. FIREY
}

1. Lebesgue [3] proved that, among all orbiforms of given breadth, the Reuleaux triangle has the least area and the circle, of course, the greatest area. Further, these are the only extremal figures. This paper is an elaboration of that result.

An orbiform is a convex body in the Euclidean plane which is such that the distance between parallel support lines (breadth) is constant. A Reuleaux polygon is an orbiform whose boundary consists of a finite number (greater than one) of circular arcs (sides). Reuleaux polygons necessarily have an odd number of sides. For details on these matters see [1]. If the sides are of equal length, the Reuleaux polygon is called regular. We shall prove that any two regular Reuleaux polygons having the same number of sides are similar. All Reuleaux triangles are regular. Our elaboration of Lebesgue's result is contained in the following three theorems.

THEORM 1. The isoperimetric ratio (ratio of area to squared perimeter) of regular Reuleaux polygons strictly increases with the number of sides.

Theorem 2. Among all Reuleaux polygons having the same number of sides, the regular Reuleaux polygons (and only these) attain the greatest isoperimetric ratio.

Theorem 3. For any odd integer $n>3$ and any $\varepsilon>0$, there is an $n$-sided Reuleaux polygon whose isoperimetric ratio exceeds that of the Reuleaux triangle by an amount less than $\varepsilon$.

As a matter of terminology, when reference is made in this paper to an $n$-sided Reuleaux polygon, it will always be understood that $n$ is odd and that none of these sides is of zero length. As a matter of notation, $|P Q|$ means the length of segment $P Q$.

2. Being concerned only with isoperimetric ratios, we limit ourselve to Reuleaux polygons of unit breadth without loss of generality. The centre $C$ from which a circular side of such a polygon is drawn must lie on the boundary of the polygon if the polygon is to be of constant breadth. Moreover, if $C^{\prime}$ and $C^{\prime \prime}$ are the end points of this side, then $C$ is the junction of those sides centred at $C^{\prime}$ and $C^{\prime \prime}$. In proceeding in a positive direction along the boundary arc strictly between $C^{\prime}$ and $C^{\prime \prime}$, the support lines of the polygon turn through an angle of measure $\phi$ equal to the length of are from $C^{\prime}$ to $C^{\prime \prime}$ which is the same as the

Received July 10, 1959. 
measure of angle $C^{\prime} C C^{\prime \prime}$. The chord $C^{\prime} C$ is perpendicular to one of the extreme support lines at $C$; the chord $C^{\prime \prime} C$ is perpendicular to the other extreme support line at $C$. Therefore the interior vertex angle at $C$ has measure $\pi-\phi$. For a regular Reuleaux polygon, it follows that these vertex angles are all equal. Conversely, if these vertex angles are all equal, the Reuleaux polygon is regular. Hence any two $n$-sided regular Reuleaux polygons are similar. Since the total turning of the support lines in one complete positive circuit of the boundary is $2 \pi$, we have, if we observe that at $C$ the turning from one extreme support line to the other is $\phi, 2 \sum_{c} \phi=2 \pi$ where the summation is carried out over all vertices $C$. This shows that the perimeter of any Reuleaux polygon of unit breadth is $\sum_{c} \phi=\pi$ and so, in comparing isoperimetric ratios of such figures, we need only to compare areas. It further shows that the sum of the measures of angles $C^{\prime} C C^{\prime \prime}$ is $\pi$.

For an $n$-sided, regular Reuleaux polygon $\phi=\pi / n$. By direct calculation, one finds that the area of such a polygon is

$$
\pi\left[1-\left(1-\cos \frac{\pi}{n}\right) /\left(\frac{\pi}{n} \sin \frac{\pi}{n}\right)\right] / 2
$$

Since

$$
\frac{d}{d \theta} \ln \frac{1-\cos \theta}{\theta \sin \theta}=\frac{1}{\sin \theta}-\frac{1}{\theta}>0
$$

for $0<\theta<\pi$, taking $\theta=\pi / n$, we see that the area is a strictly increasing function of $n$ as asserted in Theorem 1 .

3. A Reuleaux polygon $\Pi_{0}$ of unit breadth can be considered as the intersection of all closed circular discs of radius one centred at the vertices $C$ of $\Pi_{0}$. Consider those convex bodies $\Pi_{\delta}$ which are the intersection of all closed circular discs of radius $1-\delta,(\delta>0)$, centred at the vertices $C$, where $\delta$ is sufficiently small. Alternatively, $\Pi_{\delta}$ can be described as the set of centres of circles of radius greater than or equal to $\delta$ which are covered by $\Pi_{0}$. Such a body is an inner parallel of $\Pi_{0}$. It is not a body of constant breadth. Let $\delta_{0}$ be the least upper bound of the numbers $\delta$ for which $\Pi_{\delta}$ is not degenerate, (i.e. has a nonempty interior). Since the inner parallels $\Pi_{\delta}$ are intersections of closed circular discs, the degenerate parallel figure $\Pi_{\delta_{0}}$ is a point. In view of the second definition of inner parallels, this point is the centre of the inscribed circle of $\Pi_{0}$, which is of radius $\delta_{0}$. In the case of a regular Reuleaux polygon $\Pi_{0}^{\prime}$, the inscribed circle is tangent to each side at its mid-point.

Consider a vertex $C_{0}^{\prime}$ of $\Pi_{0}^{\prime}$ and the ends $C_{1}{ }^{\prime}, C_{2}^{\prime}$ of the side drawn using $C_{0}^{\prime}$ as a centre. Let $F^{\prime}$ be the intersection of circular discs of radius one centred at $C_{0}{ }^{\prime}, C_{1}{ }^{\prime}, C_{2}{ }^{\prime}$. If $\Gamma^{\prime}$ is the inscribed circle of $\Pi_{0}^{\prime}$ (of radius $\delta_{0}^{\prime}$ ), it is the inscribed circle of $F^{\prime}$. 
From $\S 2$, we know that for any Reuleaux polygon $\sum_{e} \phi=\pi$. Therefore, if $\Pi_{0}$ is an $n$-sided Reuleaux polygon which is not regular, at least one of the numbers $\phi$ must be greater than $\pi / n$. Let $C_{0}$ be a vertex at which $\phi>\pi / n$, and let $C_{1}, C_{2}, F, \Gamma$ have the same meaning relative to $\Pi_{0}$ as did $C_{1}{ }^{\prime}, C_{2}{ }^{\prime}, F^{\prime}, \Gamma^{\prime}$ relative to $I_{0}^{\prime}$. If we move $F$ so that $C_{0}$ and $C_{0}^{\prime}$ as well as $C_{1}$ and $C_{1}^{\prime}$ coincide, then $F \subset F^{\prime}$ since $\phi>\pi / n$. Now $\Gamma$ must be covered by $F$ and so, plainly, is of radius less than $\delta_{0}^{\prime}$. This gives

Lemma 1. If $I_{0}^{\prime}$ is a regular $n$-sided Reuleaux polygon, $I_{0}$ is any $n$-sided Reuleaux polygon, both being of the same breadth, and if $\delta_{0}$ is the least upper bound of the numbers for which $I_{\delta}$ is not degenerate and $\delta_{0}^{\prime}$ that for which $\Pi_{\delta}^{\prime}$ is not degenerate, then $\delta_{0} \leqq \delta_{0}^{\prime}$ with equality only only if $I_{0}$ is also regular.

Next we shall construct a formula for estimating areas of Reuleaux polygons $I I_{0}$. In all the remaining discussion $\delta \leqq \delta_{0}$. For sufficiently small $\delta$ we will have equality with the estimate, but for larger $\delta$ there will, in general, be inequality.

Let the vertices be indexed in a counter-clockwise order $C_{1}, C_{2}, \cdots, C_{n}$ and let $\phi_{i}$ be the length of the side drawn from centre $C_{i}$. Consider the set of points lying within the unit circle centred at $C_{i}$ and outside the circle of radius $1-\delta$ centred at $C_{i}$ and within the acute angular region between the rays from $C_{i}$ through the ends of the side of $\Pi_{0}$ which is drawn using $C_{i}$ as a centre. This region will be called $R_{i}(\delta)$ or simply $R_{i}$. The area of $R_{i}$ is $\delta \phi_{i}-\delta^{2} \phi_{i} / 2$.

The points of $\Pi_{0}$ which are not in at least one of these regions $R_{i}(\delta)$ make up $\Pi_{\delta}$. Let $A(S)$ denote the area of $S$. We have

$$
\begin{aligned}
& A\left(\Pi_{0}\right)=A\left(\Pi_{\delta}\right)+\sum_{i} A\left(R_{i}\right)-\sum_{i<j} A\left(R_{i} \cap R_{j}\right) \\
& \quad+\sum_{i<j<k} A\left(R_{i} \cap R_{j} \cap R_{k}\right)+\cdots+(-1)^{n+1} A\left(R_{1} \cap R_{2} \cap \cdots \cap R_{n}\right) .
\end{aligned}
$$

Empty intersections are assigned zero area. Since every intersection of $p$ of the sets $R_{i}$ is a subset of some intersection of $p-1$ of the sets $R_{i}$

$$
\begin{aligned}
\sum_{i<j<k} A\left(R_{i}\right. & \left.\cap R_{j} \cap R_{k}\right)-\sum_{i<j<k<l} A\left(R_{i} \cap R_{j} \cap R_{k} \cap R_{l}\right)+\cdots \\
& +(-1)^{n+1} A\left(R_{1} \cap R_{2} \cap \cdots \cap R_{n}\right) \leqq \sum_{i<j<k} A\left(R_{i} \cap R_{j} \cap R_{k}\right) .
\end{aligned}
$$

We next observe that

$$
\sum_{i<j} A\left(R_{i} \cap R_{j}\right)=A\left(R_{1} \cap R_{n}\right)+\sum_{i=1}^{n-1} A\left(R_{i} \cap R_{i+1}\right)+\Sigma^{\prime} A\left(R_{i} \cap R_{j}\right)
$$

where $\Sigma^{\prime}$ means the summation is extended only over indices such that $1<j-i<n-1$. We finally note that any threefold intersection $R_{i} \cap R_{j} \cap R_{k}$ must be a subset of the sets whose areas are counted in $\Sigma^{\prime} A\left(R_{i} \cap R_{j}\right)$. 
Hence we have

$$
\begin{gathered}
\sum^{\prime} A\left(R_{i} \cap R_{j}\right)-\left[\sum_{i<j<k} A\left(R_{i} \cap R_{j} \cap R_{k}\right)+\cdots\right. \\
\left.\quad+(-1)^{n+1} A\left(R_{1} \cap R_{2} \cap \cdots \cap R_{n}\right)\right] \\
\geqq \sum^{\prime} A\left(R_{i} \cap R_{j}\right)-\sum_{i<j<k} A\left(R_{i} \cap R_{j} \cap R_{k}\right) \geqq 0 .
\end{gathered}
$$

This gives, in place of equation (1), the inequality

$$
A\left(\Pi_{0}\right) \leqq A\left(\Pi_{\delta}\right)+\sum_{i} A\left(R_{i}\right)-\left[A\left(R_{1} \cap R_{n}\right)+\sum_{i=1}^{n-1} A\left(R_{i} \cap R_{i+1}\right)\right] .
$$

A last modification: the terms

$$
\sum_{i} A\left(R_{i}\right)=\sum_{i}\left(\delta \phi_{i}-\delta^{2} \phi_{i} / 2\right)=\pi \delta-\pi \delta^{2} / 2
$$

since the perimeter of $I_{0}$ is $\pi$. We have

LEMMA 2.

$$
A\left(I_{0}\right) \leqq A\left(\Pi_{\delta}\right)+\pi \delta-\pi \delta^{2} / 2-\left[A\left(R_{1} \cap R_{n}\right)+\sum_{i=1}^{n-1} A\left(R_{i} \cap R_{i+1}\right)\right]
$$

If $I_{0}$ is a regular Reuleaux polygon, there is equality for all $\delta \leqq \delta_{0}$.

The final assertion of the lemma remains to be proved. In this discussion, wherever indices greater than $n$ appear, they are to be reduced modulo $n$. When we speak of consecutive regions $R_{i}, R_{i+1}, \cdots$, $R_{i+k}$, we assume $k \leqq(n-1) / 2$.

Let $\Pi_{0}^{\prime}$ be a regular $n$-sided Reuleaux polygon. We first observe that if $R_{i}$ be rotated counterclockwise about the centre $C_{0}$ of $\Pi_{0}^{\prime}$ in the amount $2 \pi k / n$, it will coincide with $R_{i+k}$. But we can say more. Let $R_{i}^{*}$ be $R_{i} \cup S_{i}$, where $S_{i}$ is the region outside the unit circle centred at $C_{i}$ and inside the circumscribing circle of $\Pi_{0}^{\prime}$. Further, let $R_{i}^{*}(\psi)$ be the set obtained by rotating $R_{i}^{*}$ about $C_{0}$ through an angle of measure $\psi$. We reckon $\psi>0$ for counterclockwise rotation, $\psi<0$ for clockwise rotation. Our more extended result is $R_{i} \cap R_{i+k} \subseteq R_{i}^{*}(\psi) \cap R_{i+k}$ for $0 \leqq \psi \leqq 2 \pi k / n$. To see this, let $P$ be a point in $R_{i} \cap R_{i+k}$. Then $P$ is in $R_{i}^{*}(0)$ and $R_{i}^{*}(2 \pi k / n)$ which cover $R_{i}$ and $R_{i+k}$ respectively. If $P$ is not in $R_{i}^{*}\left(\psi_{1}\right),\left(0<\psi_{1}<2 \pi k / n\right)$, then there are two values of $\psi$, say $\psi_{0}$ and $\psi_{2}$, such that $P$ is on the boundary of $R_{i}^{*}\left(\psi_{0}\right)$ and $R_{i}^{*}\left(\psi_{2}\right)$ and $0<\psi_{0}<\psi_{1}<\psi_{2}<2 \pi k / n$. Let $P(\psi)$ be the image of $P$ after a rotation about $C_{0}$ in the amount $\psi$. Then $P\left(-\psi_{0}\right)$ and $P\left(-\psi_{2}\right)$ are on the boundary of $R_{i}^{*}(0)$ and $P\left(-\psi_{1}\right)$ is outside $R_{i}^{*}(0)$. A circular arc centred at $C_{0}$ is part of the circumscribing circle of $\Pi_{\delta^{\prime}}^{\prime}$ for some $\delta^{\prime}$, from which we conclude that if $P\left(-\psi_{0}\right)$ is on a circular or rectilinear part of the boundary, the same is true for $P\left(-\psi_{2}\right)$ and consequently, for all $\psi$ between $-\psi_{0}$ and $-\psi_{2}, P(\psi)$ is in the interior of $R_{i}^{*}(0)$. This contradicts the supposition that $P$ is not in $R_{i}^{*}\left(\psi_{1}\right)$. 
From this it follows that if $P$ is a point of $R_{i} \cap R_{i+k}$, it is in $R_{i+j}$ for $i \leqq j \leqq k$. For by the preceding discussion it is in $R_{i}^{*}(\psi) \cap R_{i}$ which is $R_{i+j}^{*}(0) \cap R_{i}$ for $\psi=2 \pi j / n$. Since $S_{i+j}$ and $R_{i}$ are disjoint, $P$ must be in $R_{i} \cap R_{i+j}$ as asserted.

We now consider a set $S$ of points of $\Pi_{0}^{\prime}$ which are covered by $k$ of the sets $R_{i}$. We have just shown that it must be covered by $k$ consecutive sets $R_{p}, R_{p+1}, \cdots, R_{p+k}$. In this collection there are exactly $k-1$ intersection sets $R_{i} \cap R_{i+1},(i=p, p+1, \cdots, p+k-1)$ each of which covers $S$. Thus in $\sum_{i=1}^{n} A\left(R_{i}\right)$ the area of $S$ is counted $k$ times, while in

$$
A\left(R_{1} \cap R_{n}\right)+\sum_{i=1}^{n-1} A\left(R_{i} \cap R_{i+1}\right)
$$

the area of $S$ is counted $k-1$ times. Hence the area of $S$ is counted just once in

$$
\sum_{i=1}^{n} A\left(R_{i}\right)-\left[A\left(R_{1} \cap R_{n}\right)+\sum_{i=1}^{n-1} A\left(R_{i} \cap R_{i+1}\right)\right]
$$

and so this last expression gives the area of the set of points covered by at least one of the regions $R_{i}$. The set of points of $\Pi_{0}^{\prime}$ not covered by at least one set $R_{i}$ forms $\Pi_{\delta}^{\prime}$. Therefore we have equality of areas as stated in the lemma.

Let $C$ be that common point of $R_{i}$ and $R_{i+1}$ which is a vertex of a Reuleaux polygon $\Pi_{0}$ and let $\phi$ be the measure of angle $C_{i} C C_{i+1}$. For all $\delta>0$, the sets $R_{i}(\delta) R_{i+1}(\delta)$ have an intersection of positive area. As a final preparation for Theorem 2, we shall prove

Lemma 3. For fixed $\delta$,

$$
A\left(R_{1} \cap R_{n}\right)+\sum_{i=1}^{n-1} A\left(R_{i} \cap R_{i+1}\right)
$$

is a minimum if and only if $\Pi_{0}$ is regular.

Let $Q_{i}$ be the point of intersection in $\Pi_{0}$ of the segment $C C_{i}$ with the circle of radius $1-\delta$ centred at $C_{i}$. Let $D$ be the point nearest $C$ at which circles of radius $1-\delta$ centred at $C_{i}$ and $C_{i+1}$ intersect. The set $R_{i} \cap R_{i+1}$ is symmetric about the line through $C$ and $D$. If $\rho(\theta)$ is the distance from $C$ to a point $Q$ on the circular arc of radius $1-\delta$ centred at $C_{i}$ and $\theta$ the positive measure of angle $Q C Q_{i}$, we have

$$
A\left(R_{i} \cap R_{i+1}\right)=\int_{0}^{\phi / 2} \rho^{2}(\theta) d \theta=A(\phi) .
$$

Since

$$
\rho(\theta)=\cos \theta-\sqrt{\left(\cos ^{2} \theta-2 \delta+\delta^{2}\right)} \quad \text { increases with } \theta,
$$

we have 


$$
\frac{d^{2} A(\phi)}{d \phi^{2}}=\frac{1}{2} \rho\left(\frac{\phi}{2}\right) \rho^{\prime}\left(\frac{\phi}{2}\right) \geqq 0
$$

with equality only if $\phi=0$. Therefore $A$ is a strictly convex function of $\phi$ in our considerations, since $\phi \neq 0$. From $\S 2$, we know that $\sum_{c} \phi=\pi$. Applying Jensen's inequality [2] gives

$$
n A\left(\frac{\pi}{n}\right) \leqq A\left(R_{1} \cap R_{n}\right)+\sum_{i=1}^{n-1} A\left(R_{i} \cap R_{i+1}\right)
$$

with equality if and only if each $\phi=\pi / n$.

The proof of Theorem 2 is now almost immediate. Let $\Pi_{0}$ be a Reuleaux polygon having $n$ sides and of breadth one; let $\Pi_{0}$ be a regular Reuleaux polygon of the same breadth and having the same number of sides. By Lemma 2

$$
\begin{aligned}
A\left(\Pi_{0}\right) & \leqq \delta_{0} \pi-\delta_{0}^{2} \pi / 2-\left[A\left(R_{1}\left(\delta_{0}\right) \cap R_{n}\left(\delta_{0}\right)\right)+\sum_{i=1}^{n-1} A\left(R_{i}\left(\delta_{0}\right) \cap R_{i+1}\left(\delta_{0}\right)\right)\right] \\
& \leqq \delta_{0} \pi-\delta_{0}^{2} \pi / 2-n A\left(\frac{\pi}{n}\right) \quad \text { by Lemma } 3 .
\end{aligned}
$$

From Lemma 1,

$$
A\left(\Pi_{\delta_{0}}^{\prime}\right) \geqq 0
$$

and so we have further

$$
A\left(\Pi_{0}\right) \leqq A\left(\Pi_{\delta_{0}}^{\prime}\right)+\delta_{0} \pi-\delta_{0}^{2} \pi / 2-n A\left(\frac{\pi}{n}\right)=A\left(\Pi_{0}^{\prime}\right)
$$

where the last equality is a consequence of Lemma 2 . In the first of this succession of inequalities, we can only assert there is equality if $\Pi_{0}$ is regular; but in the remaining inequalities, there is equality if and only if $\Pi_{0}$ is regular. This completes the proof of Theorem 2.

We turn to the proof of Theorem 3. Let $n>3$ and $\varepsilon$ satisfy $0<\varepsilon<\pi / 3$. Assume for the moment that it is always possible to construct a Reuleaux polygon of breadth one having one interior vertex angle of measure $2 \pi / 3+\varepsilon$. Let $C$ be such a vertex. Extend the sides which join at $C$ past their endpoints so as to meet the unit circle centred at $C$ in points $P$ and $Q$. Consider the convex body $T$ bounded by the smaller unit circular arcs $\widehat{C P}, \overparen{P Q}$ and $\widehat{Q C}$. Let its area be $A_{1}$. If $A_{0}$ is the area of the Reuleaux triangle of unit breadth, a direct computation shows $A_{1}-A_{0}=\varepsilon / 2$. By Lebesgue's theorem, if $A$ is the area of the Reuleaux polygon, $A_{0}<A$. But the body $T$ covers the Reuleaux polygon and so $0<A-A_{0}<\varepsilon$.

The preliminary assumption will be verified inductively. For this purpose we first prove

Lemma. If $P$ and $Q$ are points for which $|P Q|<1$, then it is possible to determine points $P^{\prime}$ and $Q^{\prime}$ such that $\left|P P^{\prime}\right|=\left|Q Q^{\prime}\right|=\left|P^{\prime} Q^{\prime}\right|=1$, 
$\left|P Q^{\prime}\right|<1$ and segments $P Q$ and and $P^{\prime} Q^{\prime}$ are parallel.

Choose a Cartesian coordinate system with respect to which $P$ and $Q$ have coordinates $\left(-\frac{1}{2}+\eta, 0\right)$ and $\left(\frac{1}{2}-\eta, 0\right)$ respectively. Here $0<\eta=(1-|P Q|) / 2<1 / 2$. Let $\theta=\operatorname{arc} \cos (1-\eta)$. From $P$ draw a unit segment terminating in the first quadrant at $P^{\prime}$ such that the slope of segment $P P^{\prime}$ is $\tan \theta$. Let $Q^{\prime}$ be the image of $P^{\prime}$ after reflection in the $y$-axis. $P^{\prime}$ has coordinates $\left(\frac{1}{2}, \sqrt{\left(2 \eta-\eta^{2}\right)}\right)$ and $Q^{\prime}$ has coordinates $\left(-\frac{1}{2}, \sqrt{\left(2 \eta-\eta^{2}\right)}\right)$. The assertions of the lemma can be verified directly.

To construct a Reuleaux pentagon of the desired character, let $P C$ and $Q C$ be segments of length one such that the measure of angle $P C Q$ is $\pi / 3-\varepsilon$. Then $|P Q|<1$ and we apply the construction of the lemma in such a way that points $P^{\prime}$ and $Q^{\prime}$ are on the same side of the line through $P$ and $Q$ as is $C$. The points $P, C, Q, Q^{\prime}, P^{\prime}$ are the vertices of a unit-edged star pentagon from which the desired Reuleaux pentagon can be constructed in the usual way. We remark that this Reuleaux pentagon is symmetric about the line bisecting angle $P C Q$.

Suppose that $n>3$ and $I I$ is a Reuleaux $n$-gon with an interior vertex angle of measure $2 \pi / 3+\varepsilon$ at $C$. Further, suppose $\Pi$ to be symmetric about the line $b$ bisecting this angle. Let $P_{1}$ and $Q_{1}$ be the ends of the chord of unit length which is perpendicular to $b$ and $Q_{0}$ that vertex other that $P_{1}$ which is at a unit distance from $Q_{1}$. Replace $Q_{1}$ by a point $Q$ such that, if $P$ is the symmetric image of $Q$ with respect to $b$, then angle $P Q Q_{0}$ is of greater measure than angle $P_{1} Q_{1} Q_{0}$. The choice of $Q$ is, however, restricted by requiring vertices of $I I$ which were on opposite sides of the line through $Q_{0}$ and $Q_{1}$ to be on opposite sides of the line through $Q_{0}$ and $Q$. It is easily shown that $|P Q|<\left|P_{1} Q_{1}\right|=1$. With the construction of the lemma, two points $P^{\prime}$ and $Q^{\prime}$ are constructed lying on the same side of the line through $P$ and $Q$ as $Q_{1}$. The points $P, Q, P^{\prime}, Q^{\prime}$ and vertices of $\Pi$ other than $P_{1}, Q_{1}$ from the vertices of a star polygon of $n+2$ sides all of length one. From this star an $n+2$ sided Reuleaux polygon $\Pi^{\prime}$ can be constructed. The vertex angle at $C$ appears unaltered in $I^{\prime}$ and $b$ remains a line of symmetry for $\Pi^{\prime}$. By induction, the existence of $n$-sided Reuleaux polygons having an interior vertex angle of measure $2 \pi / 3+\varepsilon$ is established.

\section{REFERENCE}

1. T. Bonnesen, and W. Fenchel, Theorie der Konvexen Körper. N Y, (1948), 130-135.

2. G. Hardy, J. Littlewood, and G. Pólya, Inequalities. Cambridge, (1934), 70-77.

3. H. Lebesgue, Sur le problème des isopérimètres et sur les domaines de largeur constante, Bull. Soc. Math. France C. R. (1914), 72-76.

State College of Washington 



\section{PACIFIC JOURNAL OF MATHEMATICS}

\section{EDITORS}

\author{
David Gilbarg \\ Stanford University \\ Stanford, California \\ F. H. Brownell \\ University of Washington \\ Seattle 5 , Washington
}

\author{
A. L. Whiteman \\ University of Southern California \\ Los Angeles 7, California \\ L. J. PAIGE \\ University of California \\ Los Angeles 24, California
}

\section{ASSOCIATE EDITORS}

\author{
E. F. BECKENBACH \\ T. M. CHERRY \\ D. DERRY
}
E. HEWITT
A. HORN
L. NACHBIN
M. OHTSUKA
H. L. ROYDEN
M. M. SCHIFFER

E. SPANIER

E. G. STRAUS

F. WOLF

\section{SUPPORTING INSTITUTIONS}

\author{
UNIVERSITY OF BRITISH COLUMBIA \\ CALIFORNIA INSTITUTE OF TECHNOLOGY \\ UNIVERSITY OF CALIFORNIA \\ MONTANA STATE UNIVERSITY \\ UNIVERSITY OF NEVADA \\ NEW MEXICO STATE UNIVERSITY \\ OREGON STATE COLLEGE \\ UNIVERSITY OF OREGON \\ OSAKA UNIVERSITY \\ UNIVERSITY OF SOUTHERN CALIFORNIA
}

\author{
STANFORD UNIVERSITY \\ UNIVERSITY OF TOKYO \\ UNIVERSITY OF UTAH \\ WASHINGTON STATE COLLEGE \\ UNIVERSITY OF WASHINGTON

AMERICAN MATHEMATICAL SOCIETY
CALIFORNIA RESEARCH CORPORATION
HUGHES AIRCRAFT COMPANY
SPACE TECHNOLOGY LABORATORIES
NAVAL ORDNANCE TEST STATION

Mathematical papers intended for publication in the Pacific Journal of Mathematics should be typewritten (double spaced), and the author should keep a complete copy. Manuscripts may be sent to any one of the four editors. All other communications to the editors should be addressed to the managing editor, L. J. Paige at the University of California, Los Angeles 24, California. ..

50 reprints per author of each article are furnished free of charge; additional copies may be obtained at cost in multiples of 50 .

The Pacific Journal of Mathematics is published quarterly, in March, June, September, and December. The price per volume (4 numbers) is $\$ 12.00$; single issues, $\$ 3.50$. Back numbers are available. Special price to individual faculty members of supporting institutions and to individual members of the American Mathematical Society: $\$ 4.00$ per volume; single issues, $\$ 1.25$.

Subscriptions, orders for back numbers, and changes of address should be sent to Pacific Journal of Mathematics, 2120 Oxford Street, Berkeley 4, California.

Printed at Kokusai Bunken Insatsusha (International Academic Printing Co., Ltd.), No. $6_{4}$ 2-chome, Fujimi-cho, Chiyoda-ku, Tokyo, Japan.

PUBLISHED BY PACIFIC JOURNAL OF MATHEMATICS, A NON-PROFIT CORPORATION

The Supporting Institutions listed above contribute to the cost of publication of this Journăl, but they are not owners or publishers and have no responsibility for its content or policies. 


\section{Pacific Journal of Mathematics}

\section{Vol. 10, No. $3 \quad$ November, 1960}

Glen Earl Baxter, An analytic problem whose solution follows from a simple

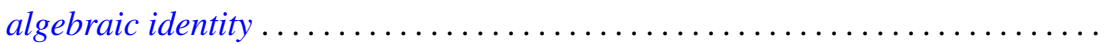

Leonard D. Berkovitz and Melvin Dresher, A multimove infinite game with linear payoff. .

Earl Robert Berkson, Sequel to a paper of A. E. Taylor ......................

Gerald Berman and Robert Jerome Silverman, Embedding of algebraic systems.... 767

Peter Crawley, Lattices whose congruences form a boolean algebra . . . . . ...... 777

Robert E. Edwards, Integral bases in inductive limit spaces . . . . . . . . . . . . . . .

Daniel T. Finkbeiner, II, Irreducible congruence relations on lattices . . . . . . . . . .

William James Firey, Isoperimetric ratios of Reuleaux polygons . . . . . . . . . . . 787

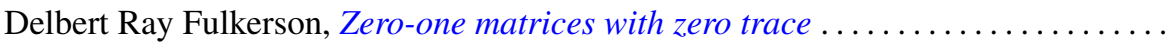

Leon W. Green, A sphere characterization related to Blaschke's conjecture........

Israel (Yitzchak) Nathan Herstein and Erwin Kleinfeld, Lie mappings in

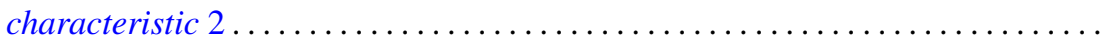

Charles Ray Hobby, A characteristic subgroup of a $p$-group .................

R. K. Juberg, On the Dirichlet problem for certain higher order parabolic

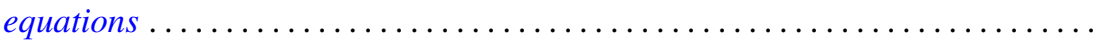

Melvin Katz, Infinitely repeatable games ......................

Emma Lehmer, On Jacobi functions . . . . . . . . . . . . . . . . . . . . . . . . .

D. H. Lehmer, Power character matrices

Henry B. Mann, A refinement of the fundamental theorem on the density of the sum of two sets of integers.

Marvin David Marcus and Roy Westwick, Linear maps on skew symmetric matrices: the invariance of elementary symmetric functions . .

Richard Dean Mayer and Richard Scott Pierce, Boolean algebras with ordered

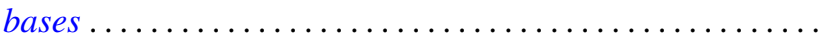

Trevor James McMinn, On the line segments of a convex surface in $E_{3} \ldots$

Frank Albert Raymond, The end point compactification of manifolds ..

Edgar Reich and S. E. Warschawski, On canonical conformal maps of regions of arbitrary connectivity

Marvin Rosenblum, The absolute continuity of Toeplitz's matrices...

Lee Albert Rubel, Maximal means and Tauberian theorems . .

Helmut Heinrich Schaefer, Some spectral properties of positive linear operators

Jeremiah Milton Stark, Minimum problems in the theory of pseudo-conformal transformations and their application to estimation of the curvature of the invariant metric.

Robert Steinberg, The simplicity of certain groups ...

Hisahiro Tamano, On paracompactness. .

Angus E. Taylor, Mittag-Leffler expansions and spectral theory .

Marion Franklin Tinsley, Permanents of cyclic matrices ...... . 